\title{
Heart failure determines the myocardial inflammatory response to injury
}

\author{
Kirsten A. Kortekaas ${ }^{1 *}$, Jan H. Lindeman², Michel I. Versteegh', Els van Beelen ${ }^{3}$, \\ Robert Kleemann ${ }^{4}$, and Robert J. Klautz ${ }^{1}$
}

\begin{abstract}
${ }^{1}$ Department of Cardiothoracic Surgery, Leiden University Medical Center, 2300 RC Leiden, The Netherlands; ${ }^{2}$ Department of General Surgery Leiden University Medical Center, Leiden, The Netherlands; ${ }^{3}$ Department of Immunohematology and Blood Transfusion, Leiden University Medical Center, Leiden, The Netherlands; and ${ }^{4}$ Department of Vascular and Metabolic Diseases, TNO-Quality of Life, Leiden, The Netherlands
\end{abstract}

Received 25 March 2012; revised 9 September 2012; accepted 12 October 2012; online publish-ahead-of-print 9 November 2012

\begin{abstract}
Aims
Systemic complications after cardiac surgery are common in heart failure patients. However, the pathophysiological mechanisms, such as a different local inflammatory response of failing hearts, remain in question. This study examines whether failing hearts respond differently to cardioplegic arrest and reperfusion compared with non-failing hearts (controls).

Methods and results

The inflammatory response was evaluated in samples collected simultaneously from the radial artery and coronary sinus, and myocardial tissue in 62 patients undergoing cardiac surgery. No myocardial release of inflammatory mediators was observed upon reperfusion in controls $(n=19)$. In contrast, in patients with heart failure, reperfusion was characterized by a myocardial release of several cytokines. Myocardial interleukin- 6 was $115 \%$ increased in non-ischaemic heart failure $(n=18, P=0.002)$ as compared with a $117 \%$ increase in patients with ischaemic heart failure $(n=25, P=0.01)$. Furthermore, a myocardial release of monocyte chemoattractant protein-1 was observed in both patient groups: a 109\% $(P=0.001)$ and $114 \%(P=0.01)$ increase in patients with non-ischaemic heart failure and ischaemic heart failure, respectively. Post-operative myocardial damage, expression of inflammatory mediators, and $\mathrm{p} 65$-nuclear factor- $\mathrm{k}$ activity were similar in all patient groups. Inflammatory cell content was increased in early ischaemic myocardial tissue in both heart failure groups compared with controls.
\end{abstract}

Conclusion

Heart failure patients show a clear myocardial inflammatory response upon reperfusion, probably explained by degranulation of infiltrated inflammatory cells. Results in controls indicate that they better withstand cardioplegic arrest and reperfusion without an explicit myocardial inflammatory response.

Keywords

\section{Introduction}

Systemic complications after cardiac surgery, such as vasoplegia and infections, are common in patients with pre-existing heart failure. These complications are a major contributor to morbidity and mortality. A remarkable observation is that these systemic complications appear to be independent of their pump failure itself. ${ }^{1}$ They merely seem to reflect a different response of the body to the surgical procedure. This response can be due to systemic alterations in the setting of heart failure or may reflect a different response of the failing heart to cardioplegic arrest and reperfusion.
Ischaemia/reperfusion (I/R) injury is the paradoxical increase of tissue damage upon reperfusion of ischaemic tissue. This injury is an inherent component of cardiac surgery with artificial arrest and subsequent myocardial reperfusion. The pathophysiology of myocardial I/R injury is complex and not fully elucidated yet. Animal studies show that a complex cascade of events ultimately result in a myocardial inflammatory response upon reperfusion. ${ }^{2-4}$ Whether this can be extrapolated to the more complex situation in humans is unknown.

In this clinical study, we evaluated whether failing hearts respond differently to cardioplegic arrest and reperfusion compared with non-failing hearts. The inflammatory response was studied in the

* Corresponding author. Tel: +3171526 3445, Fax: +31 71526 6899, Email: k.a.kortekaas@lumc.nl

Published on behalf of the European Society of Cardiology. All rights reserved. (C) The Author 2012. For permissions please email: journals.permissions@oup.com. 
two most common types of heart failure, non-ischaemic and ischaemic heart failure, and compared with patients without heart failure undergoing cardiac surgery. Release of various inflammatory mediators was sequentially measured after myocardial reperfusion in human cardiac surgery. Selective arteriovenous concentration differences could be obtained directly over the reperfused heart by cannulation of the coronary sinus (i.e. the venous pool of the heart). We evaluated which factors were released from the myocardium and whether failing hearts differ from nonfailing hearts in their local inflammatory responses.

\section{Methods}

\section{Patient population}

In this 2-year prospective study, 62 patients were included: 43 patients with heart failure and 19 controls (Supplementary material, Figure S1). All heart failure patients were scheduled for restrictive mitral annuloplasty (RMA) ring implantation. Patients with non-ischaemic heart failure $(n=18)$ also underwent a concomitant implantation of an external cardiac support device (CorCap, Acorn Cardiovascular Inc., St Paul, MN, USA). Patients with ischaemic heart failure $(n=25)$ underwent coronary artery bypass surgery $(n=14)$ or ventricular reconstruction surgery if they had a large area of anterior akinesia or dyskinesia $(n=11)$ in addition to RMA. Patients without heart failure, scheduled for reconstructive mitral valve surgery for degenerative, rheumatic, or cured endocarditis mitral valve pathology, were selected as controls. These controls were expected to have approximately the same aortic cross-clamp times as heart failure patients. Heart failure was pre-operatively defined as an inadequate pump function of the heart with an echocardiographically estimated ejection fraction biplane below $35 \%{ }^{5}$ and the presence of one or more clinical symptoms as classified by the New York Heart Association (NYHA). All controls had a moderate to normal pump function of the heart (defined as an ejection fraction biplane $>45 \%$ ), no clinical signs of heart failure by physical examination, and did not use high doses of diuretics. Exclusion criteria were perioperative corticosteroid treatment, active infection, minimal invasive surgical procedures, emergency operations, and previous cardiac surgery. Patients were followed during total hospital stay, including hospital stay after discharge to a referring hospital. This study was carried out in accordance with the Declaration of Helsinki and was approved by the local ethics committee. All patients provided written informed consent.

\section{Anaesthesia and surgical procedures}

All participating patients received standardized anaesthetic procedures, according to a fast-track protocol. A 5 French indwelling jugular vein catheter (PICC, Arrow International Inc., REF PS-01651, USA) was inserted into the right atrium and placed in the coronary sinus by the surgeon. Since all patients underwent mitral valve surgery using a vertical trans-septal incision, the coronary sinus could be easily cannulated during the surgical procedure. An arterial catheter was routinely placed in the radial artery. Cardiac surgery was performed according to local standardized protocols. All surgical procedures were performed via a midline sternotomy under normothermic cardiopulmonary bypass (CPB; Jostra Maquet, Maquet, Hirrlingen, Germany) with intermittent antegrade warm-blood cardioplegia and heparin softline coating. A uniform CPB protocol was designed, excluding autologous priming and ultrafiltration.

\section{Plasma measurements}

\section{Arteriovenous measurements}

Arterial (radial artery) and myocardial venous blood samples (coronary sinus) were collected simultaneously over the reperfused heart (Figure 1). With this approach, accurate and specific measurements of locally ongoing processes were assessed up to $24 \mathrm{~h}$ after reperfusion. Arterial and myocardial venous blood samples were obtained during the early reperfusion phase: $0,15,30$, and $60 \mathrm{~min}$ after the start of reperfusion, i.e. after removing the aortic cross-clamp (early phase), and at 2, 4, 8, and $24 \mathrm{~h}$ after reperfusion (late phase). In all patients, a baseline sample was taken the day before surgery from the brachial vein. All samples were collected in pre-cooled tubes containing EDTA (BD Vacutainer, Plymouth, UK) and immediately placed on melting ice. Blood samples were centrifuged within $1 \mathrm{~h}$ after collection $\left(1550 \mathrm{~g}, 10 \mathrm{~min}, 4^{\circ} \mathrm{C}\right.$ ) and the derived plasma was re-centrifuged $\left(10000 \mathrm{~g}, 4 \mathrm{~min}, 4^{\circ} \mathrm{C}\right)$ to obtain leucocyte- and thrombocyte-free plasma. Aliquots were stored at $-70^{\circ} \mathrm{C}$ until analysis.

\section{Measurement of inflammatory mediators}

A pilot study was performed in five heart failure patients (three patients with ischaemic heart failure and two patients with nonischaemic heart failure) and five controls to identify detectable inflammatory mediators with the sensitive human 27-plex panel (Biorad, Veenendaal, The Netherlands). Based on the results of this pilot study, the following 12 cytokines were measured in the complete cohort with a custom-made multiplex assay according to the instructions of the manufacturer (X-plex, Biorad): interleukin-1 receptor antagonist (IL-1ra), IL-6, IL-8, IL-9, IL-10, IL-16, IL-18, granulocyte colony-stimulating factor (G-CSF), interferon-inducible protein-10 (IP-10), monocyte chemoattractant protein-1 (MCP-1), regulated upon activation, normal $T$ cell expressed and secreted (RANTES), and vascular endothelial growth factor (VEGF). Underlying mechanisms for the myocardial release of inflammatory mediators were investigated in the complete cohort. First, the possibility of passive secretion from damaged myocytes was looked for by measurement of troponin T. Next, we investigated whether active production and secretion by myocardial cells might be responsible for the release of inflammatory

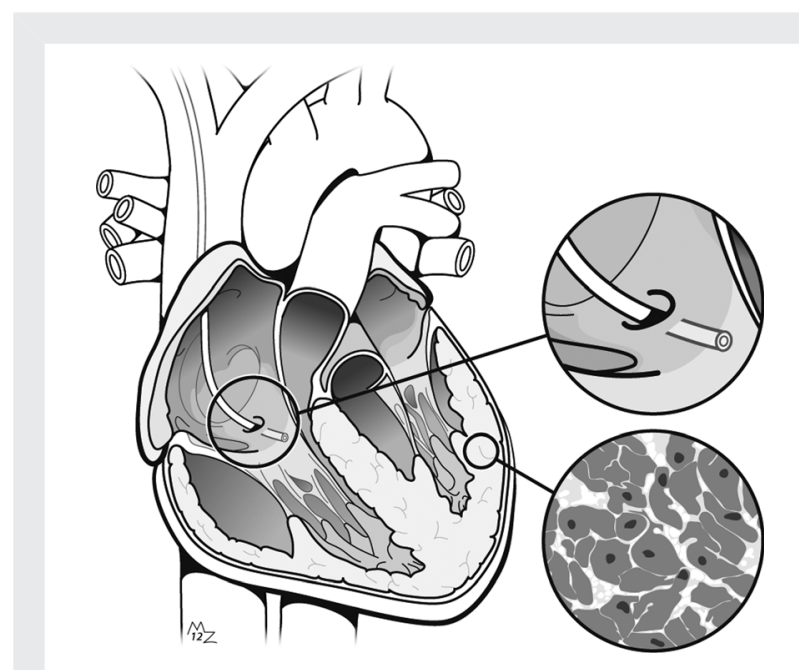

Figure I Schematic representation of the coronary sinus catheter and location of the biopsies. This figure shows the position of the coronary sinus catheter and the location of the left ventricular biopsies. ${ }^{\circ}$ ManonProject.com 
mediators by means of mRNA expression of various inflammatory mediators. Moreover, cellular activation was examined by assessing transcriptionally active nuclear factor- $\mathrm{kB}$ (NF- $\mathrm{kB}$ ). Eventually, the possibility of release by infiltrating inflammatory cells was investigated.

\section{Myocardial damage}

To assess myocardial damage, troponin $T$ values were measured by a routine laboratory assay (Roche Diagnostics, Mannheim, Germany). Dissimilarity in myocardial damage between the groups could explain differences in the myocardial release of inflammatory mediators. Troponin $\mathrm{T}$ values were measured $45 \mathrm{~min}$ after reperfusion in coronary sinus blood. This time point lies within the range of the early reperfusion phase, where the most dominant myocardial release of inflammatory mediators was observed. After cardiac valve surgery, troponin $\mathrm{T}$ values $<2.0 \mu \mathrm{g} / \mathrm{L}$ are considered normal.

\section{Myocardial tissue samples}

Through the mitral valve, an endomyocardial biopsy was obtained from a standardized location at the posterior wall of the left ventricle between the two papillary muscles, early after aortic cross-clamping. The late endomyocardial biopsy was collected on average $70 \mathrm{~min}$ later from the same location just before release of the aortic crossclamp (Figure 1). Biopsies were divided and fixed in formalin or immediately snap-frozen in liquid nitrogen and stored at $-70^{\circ} \mathrm{C}$. Formalinfixed paraffin-embedded biopsies were cut into $5 \mu \mathrm{m}$ thick sections with a microtome.

\section{RNA extraction and mRNA analysis}

Total RNA was prepared from early and late endomyocardial biopsies. Total RNA extraction was performed using RNA-Bee (Campro Scientific, Veenendaal, The Netherlands) and glass beads. Subsequently, cDNA was prepared using a High Capacity RNA-to-cDNA Kit (Applied Biosystems Inc., Foster City, CA, USA). Taqman gene expression master mix, ABI 7500 Fast system, and established primer/probe sets (Applied Biosystems Inc.) were used according to the manufacturer's instructions to quantify IL-1ra (Hs00893626_m1), IL-6 (Hs00174131_m1), IL-8 (Hs00174103_m1), IL-10 (Hs00961622_m1), tumour necrosis factor (TNF)- $\alpha$ (Hs00174128_m1), IL-1 $\beta$ (Hs00174097_m1), matrix metallopeptidase (MMP)-9 (Hs00957562_m1), cluster of differentiation (CD)68 (Hs00154355_m1), and MCP-1 (Hs00234140_m1). Glyceraldehyde phosphate dehydrogenase (GAPDH; VIC labelled) was used as housekeeping gene (Applied Biosystems Inc.).

Analysis of intramyocardial p65-nuclear factor- $\kappa B$ activity To assess the role of NF- $\mathrm{KB}$ as a transcription factor controlling the expression of genes involved in immune responses, $\mathrm{p} 65-\mathrm{NF}-\kappa \mathrm{B}$ activity was quantified in paired early and late endomyocardial biopsies of five patients of each patient group. Myocardial p65-NF-кB activity was determined as described previously by Kleemann et al., ${ }^{6}$ using a TransAM transcription factor assay kit (no. 40097, Active Motif Europe, Rixensart, Belgium). The assays were performed according to the instructions of the manufacturer. Myocardial tissue homogenates were prepared using the Nuclear Extract Kit (no. 40010, Active Motif Europe). Equal amounts of protein $(6 \mu \mathrm{g} /$ well) of these homogenates were used to determine the amount of active p65-NF-кB.

\section{Immunohistochemical analysis of left ventricular tissue}

Formalin-fixed, paraffin-embedded microtome sections were deparaffinized and rehydrated before staining for CD3, CD68, myeloperoxidase (MPO), and human leucocyte antigen (HLA)-DR. Some stainings required the use of a protein retrieval: an EDTA buffer solution for CD3 and CD68 ( $\mathrm{pH}=9.0)$, and a citrate buffer solution $(\mathrm{pH}=6.0)$ for HLA-DR. Sections were incubated overnight with the primary antibody raised against CD3 (1:200, Abcam, Cambridge, MA, USA), CD68 (1:4000, DAKO, Glostrup, Denmark), MPO (1:5000, DAKO), and HLA-DR (1:200, DAKO) for staining of T lymphocytes, macrophages, polymorphonuclear neutrophils, and activated leucocytes, respectively. Subsequently, envision and 3,3' diaminobenzidine tetrahydrochloride as a chromogen for detection were applied. Slides were counterstained with Mayer's haematoxylin to allow morphological analysis. The number of positive cells was quantified in five views (original magnifications $\times 200$; AxioVision software, version 4.4.1.0 Carl Zeiss Microlmaging Inc., Gottingen, Germany). The number of cells over five calibrated grid areas per view was counted using Imagej Cell Counter ${ }^{\circledR}$ by an independent blinded observer.

\section{Statistical analysis}

Continuous variables were analysed using the Kruskal-Wallis test with post-hoc analyses with Mann-Whitney U-tests whenever appropriate. The significant Kruskal-Wallis offers protection against an inflated $\alpha$-error rate when performing multiple comparisons. Furthermore, Benjamini-Hochberg correction was used to correct for multiple comparisons. If values were no longer significant after this correction, NS was placed in the text after the unadjusted $P$-value. The area under the curve (AUC) was calculated for the arterial and venous curves of the plasma measurements for the early and late reperfusion phase. A delta AUC was calculated (venous minus arterial) and the null hypothesis (delta AUC is 0 ) was tested by a paired $t$-test. Histological stainings were analysed with a one-way between-groups analysis of variance (ANOVA). The Wilcoxon signed rank test was used to analyse differences between early and late ischaemic biopsies for the mRNA analysis and NF- $\mathrm{KB}$ activity. All statistical tests were performed using SPSS statistical analysis software version 17.0 (SPSS Inc., Chicago, IL, USA). A $P$-value $<0.05$ was considered significant. Graph error bars represent the mean \pm standard error of the mean (SEM) unless stated otherwise.

\section{Results}

\section{General findings}

Patient characteristics are summarized in Table 1. A similar gender distribution was observed in all groups $(P=0.43)$. In two controls, a mitral valve replacement had to be done; a repair was not possible due to extensive calcifications. Moreover, one patient was scheduled for a mitral valve repair in combination with a left ventricular reconstruction procedure. Although included, this patient did not undergo this valve repair since there was no longer any mitral regurgitation after the reconstruction procedure. Two patients died during follow-up in hospital: one patient with nonischaemic heart failure died unexpectedly 8 days after surgery as a result of ventricular fibrillation. The other patient, with ischaemic heart failure, died 41 days after ventricular reconstruction surgery as a result of therapy-resistant heart failure. Baseline levels of all inflammatory mediators were comparable in all patient groups (Supplementary material, Table S1), of which controls generally showed the lowest baseline levels of pro-inflammatory mediators. Systemic levels of inflammatory mediators, i.e. concentrations in arterial blood samples, were similar in the first hour after reperfusion in all groups except for IL-1ra, IL-6, and G-CSF (Supplementary material, Figure S2). 
Table I Baseline, surgical, and outcome characteristics of patients with non-ischaemic heart failure, ischaemic heart failure, and controls

\begin{tabular}{|c|c|c|c|c|c|c|c|}
\hline & \multicolumn{3}{|c|}{ Median (interquartile range) } & \multirow[b]{2}{*}{$\begin{array}{l}\text { Kruskal-Wallis, } \\
P \text {-value }\end{array}$} & \multicolumn{3}{|c|}{ Mann-Whitney, $P$-value } \\
\hline & NIHF $(n=18)$ & IHF $(n=25)$ & Co $(n=19)$ & & NIHF vs. IHF & IHF vs. Co & NIHF vs. Co \\
\hline Age (years) & $64.56(57.58-70.11)$ & $66.86(58.04-73.13)$ & $64.28(55.45-75.46)$ & 0.55 & - & - & - \\
\hline NYHA class & $3(3-3)$ & $3(3-3)$ & $2(1-2)$ & $<0.001$ & 0.28 & $<0.001 *$ & $<0.001 *$ \\
\hline Systolic blood pressure $(\mathrm{mmHg})$ & $108(99-116)$ & $120(110-130)$ & $125(120-145)$ & 0.001 & $0.01 *$ & 0.14 & $<0.001^{*}$ \\
\hline Diastolic blood pressure $(\mathrm{mmHg})$ & $65(60-70)$ & $70(65-85)$ & $76(70-80)$ & 0.005 & 0.04 (NS) & 0.22 & $0.001 *$ \\
\hline Body mass index $\left(\mathrm{kg} / \mathrm{m}^{2}\right)$ & $27.91(24.58-30.14)$ & $27.16(25.02-31.86)$ & $26.22(24.44-27.44)$ & 0.18 & - & - & - \\
\hline \multicolumn{8}{|l|}{ Co-morbidities ( $n$ ) } \\
\hline Diabetes & 8 & 3 & 1 & & & & \\
\hline COPD & 4 & 3 & 2 & & & & \\
\hline Atrial fibrillation & 5 & 5 & 5 & & & & \\
\hline Logistic EuroSCORE (\%) & $10.70(5.64-16.65)$ & $6.96(5.23-11.46)$ & $3.07(1.51-4.25)$ & $<0.001$ & 0.47 & $<0.001 *$ & $<0.001 *$ \\
\hline \multicolumn{8}{|l|}{ Laboratory tests } \\
\hline NT-proBNP (ng/L) & $1960.00(876.68-2858.25)$ & $1663.00(517.65-2650.50)$ & $853.40(106.85-1159.5)$ & 0.004 & 0.28 & $0.02 *$ & $0.002 *$ \\
\hline Haemoglobin (mmol/L) & $8.8(8.0-9.5)$ & $8.6(8.1-9.1)$ & $9.0(8.5-9.5)$ & 0.37 & - & - & - \\
\hline Creatinine $(\mu \mathrm{mol} / \mathrm{L})$ & $90(73-113)$ & $89(74-111)$ & $85(77-108)$ & 0.93 & - & - & - \\
\hline $\mathrm{CRP}(\mathrm{mg} / \mathrm{L})$ & $<3(<3-5.3)$ & $<3(<3-4.0)$ & $<3(<3-3.5)$ & 0.50 & - & - & - \\
\hline \multicolumn{8}{|l|}{ Echocardiography } \\
\hline LVEF biplane (\%) & $30(24-32)$ & $31(27-35)$ & $53(49-60)$ & $<0.001$ & 0.19 & $<0.001^{*}$ & $<0.001 *$ \\
\hline LVESV (mL) & $147(114-176)$ & $148(107-156)$ & $59(48-71)$ & $<0.001$ & 0.51 & $<0.001 *$ & $<0.001 *$ \\
\hline LVEDV (mL) & $202(167-245)$ & $208(167-223)$ & $135(117-146)$ & $<0.001$ & 0.85 & $<0.001 *$ & $<0.001 *$ \\
\hline \multicolumn{8}{|l|}{ Use of medication (n) } \\
\hline ACE inhibitors/ARBs & 16 & 23 & 11 & & & & \\
\hline Beta-blockers & 16 & 24 & 10 & & & & \\
\hline Statins & 14 & 20 & 6 & & & & \\
\hline Diuretics & 18 & 21 & 9 & & & & \\
\hline Digoxin & 5 & 1 & 1 & & & & \\
\hline Aortic cross-clamp time (min) & $88(83-109)$ & $151(130-192)$ & $125(105-177)$ & $<0.001$ & $<0.001 *$ & 0.14 & $0.005^{*}$ \\
\hline Ventilation time (h) & $12(7-24)$ & $14(8-24)$ & $6(6-11)$ & 0.02 & 0.68 & $0.006 *$ & 0.045 (NS) \\
\hline APACHE IV score & $52(46-61)$ & $57(48-66)$ & $42(28-57)$ & 0.04 (NS) & 0.58 & $0.02 *$ & 0.06 \\
\hline Total stay in ICU (h) & $67(23-91)$ & $42(23-114)$ & $22(20-24)$ & 0.002 & 0.89 & $0.001 *$ & $0.004^{*}$ \\
\hline Total stay in hospital (days) & $14(11-21)$ & $15(12-21)$ & $13(10-17)$ & 0.39 & - & - & - \\
\hline
\end{tabular}




\section{Myocardial release of inflammatory mediators in patients with pre-existing heart failure}

Arteriovenous differences were used to determine the myocardial cytokine and chemokine release upon reperfusion. The difference in the AUC of the arterial and venous curves was assessed to quantify the net release from the myocardium. Our analysis method is illustrated in Figure 2. Both heart failure groups showed a significant myocardial release of various cytokines upon early reperfusion. As shown in Figure 3, early myocardial reperfusion of patients with non-ischaemic heart failure was associated with a release of IL-1ra $(P=0.04$, NS), IL-6 $(P=0.002)$, IL-10 $(P=0.002)$, G-CSF $(P=0.02)$, MCP-1 $(P=0.001)$, and IL-18 $(P=0.04$, NS). In patients with ischaemic heart failure, early reperfusion was characterized by a more limited number of inflammatory markers that were released: IL-6 ( $P=0.01)$, MCP-1 $(P=0.01)$, and VEGF $(P=0.004)$. This inflammatory response

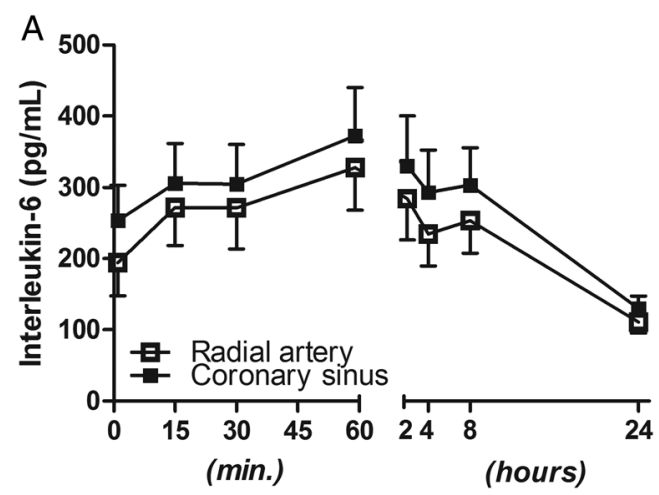

Time after reperfusion

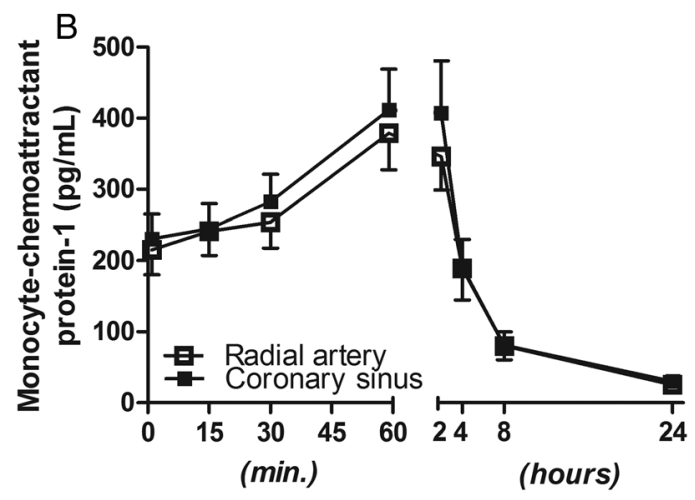

Time after reperfusion

Figure 2 Illustration of our analysis method in patients with non-ischaemic heart failure. Arteriovenous differences were used to determine the release of inflammatory markers upon reperfusion. The difference in the area under the curve of the arterial and myocardial venous curves was assessed to quantify the net release from the myocardium. A significant myocardial release of interleukin-6 as well as monocyte chemoattractant protein-1 was observed in patients with non-ischaemic heart failure upon reperfusion. Graph error bars represent mean \pm SEM. was not observed during the late reperfusion phase. The early cytokine response was exclusive for heart failure patients, since controls did not show a significant myocardial release of any of the measured inflammatory mediators upon early and late reperfusion.

\section{Similar post-operative myocardial damage}

To assess whether variation in myocardial damage is the cause of the observed differences in cytokine release between the groups, troponin T values were measured $45 \mathrm{~min}$ after reperfusion. No differences were observed between the patient groups $(P=0.67)$. The median and interquartile range of troponin $T$ was $1.24 \mu \mathrm{g} / \mathrm{L}(0.93-1.49)$ in patients with non-ischaemic heart failure, $1.36 \mu \mathrm{g} / \mathrm{L}(0.83-2.49)$ in patients with ischaemic heart failure, and $1.25 \mu \mathrm{g} / \mathrm{L}(0.83-1.60)$ in controls. A number of patients with ischaemic heart failure underwent a ventriculotomy in the setting of their surgical ventricular reconstruction which might explain the higher range of troponin $T$ values.

\section{Similar expression of inflammatory mediators during ischaemia}

A subsequent analysis of inflammatory mediators in mRNA extracts of endomyocardial tissue showed no differences at baseline in all groups (early endomyocardial biopsies, Supplementary material, Table S2), except for a small difference in MMP-9 expression in patients with non-ischaemic heart failure and controls $(P=$ 0.01). More importantly, there were no differences in inflammatory gene expression in early vs. late endomyocardial biopsies in all patient groups (Supplementary material, Table S3). This indicates that transcriptional changes are not responsible for the increased cytokine release in heart failure patients.

\section{Similar p65-nuclear factor- $\kappa B$ activity during ischaemia}

Since transcription of cytokines itself did not change, NF- $\mathrm{kB}$ was measured as many of the myocardial released inflammatory mediators are under transcriptional control of this regulator. Myocardial NF- $\mathrm{KB}$ activity was compared between the groups using homogenates of paired endomyocardial tissue samples collected in the early and late ischaemic period. Baseline values of NF- $\mathrm{KB}$ activity were similar between the groups $(P=1.00)$. No increase in NF- $\kappa \mathrm{B}$ activity was observed in early vs. late endomyocardial biopsies $(P=0.23, P=0.14$, and $P=0.89$ for patients with non-ischaemic heart failure, ischaemic heart failure, and controls, respectively; Supplementary material, Figure S3).

\section{More tissue macrophages and neutrophils in heart failure patients}

Immunohistochemical stainings were performed to evaluate whether differences in inflammatory cell content caused differences in cytokine release between the groups. There was a significant difference in CD68-positive and MPO-positive cells between the groups in the early biopsies (Figure 4A). Post-hoc comparisons indicated that the amount of macrophages was 3.8 times higher in patients with non-ischaemic heart failure relative to controls 


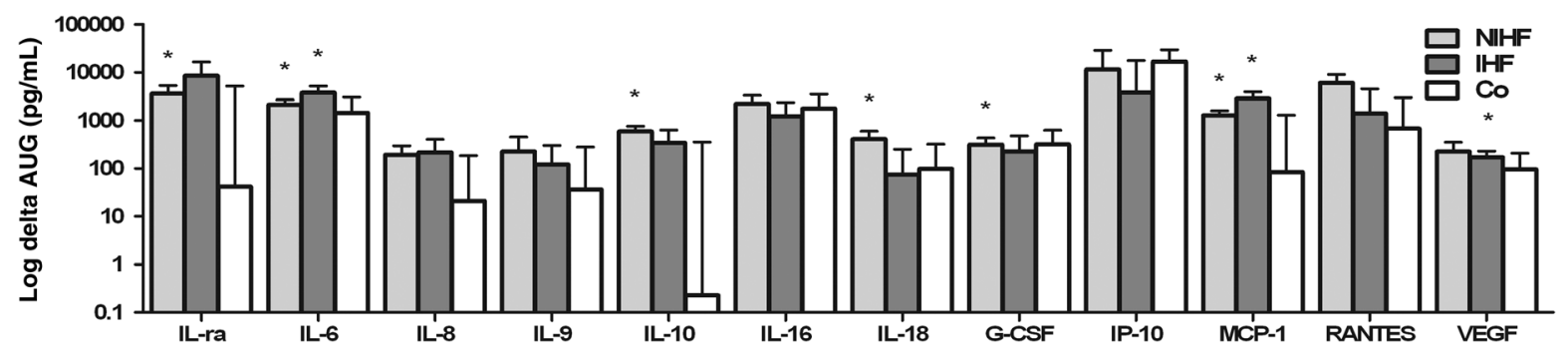

Figure 3 Myocardial release of inflammatory mediators in patients with heart failure. Various inflammatory mediators were released by the myocardium in patients with non-ischaemic (NIHF) and ischaemic heart failure (IHF) upon early reperfusion. No significant myocardial release was observed in controls (Con). Graph error bars represent mean \pm SEM. IL-1ra, interleukin-1 receptor antagonist; IL-6, interleukin 6; G-CSF, granulocyte colony-stimulating factor; IP-10, interferon-inducible protein-10; MCP-1, monocyte chemoattractant protein-1; RANTES, regulated upon activation, normal T cell expressed and secreted; VEGF, vascular endothelial growth factor.

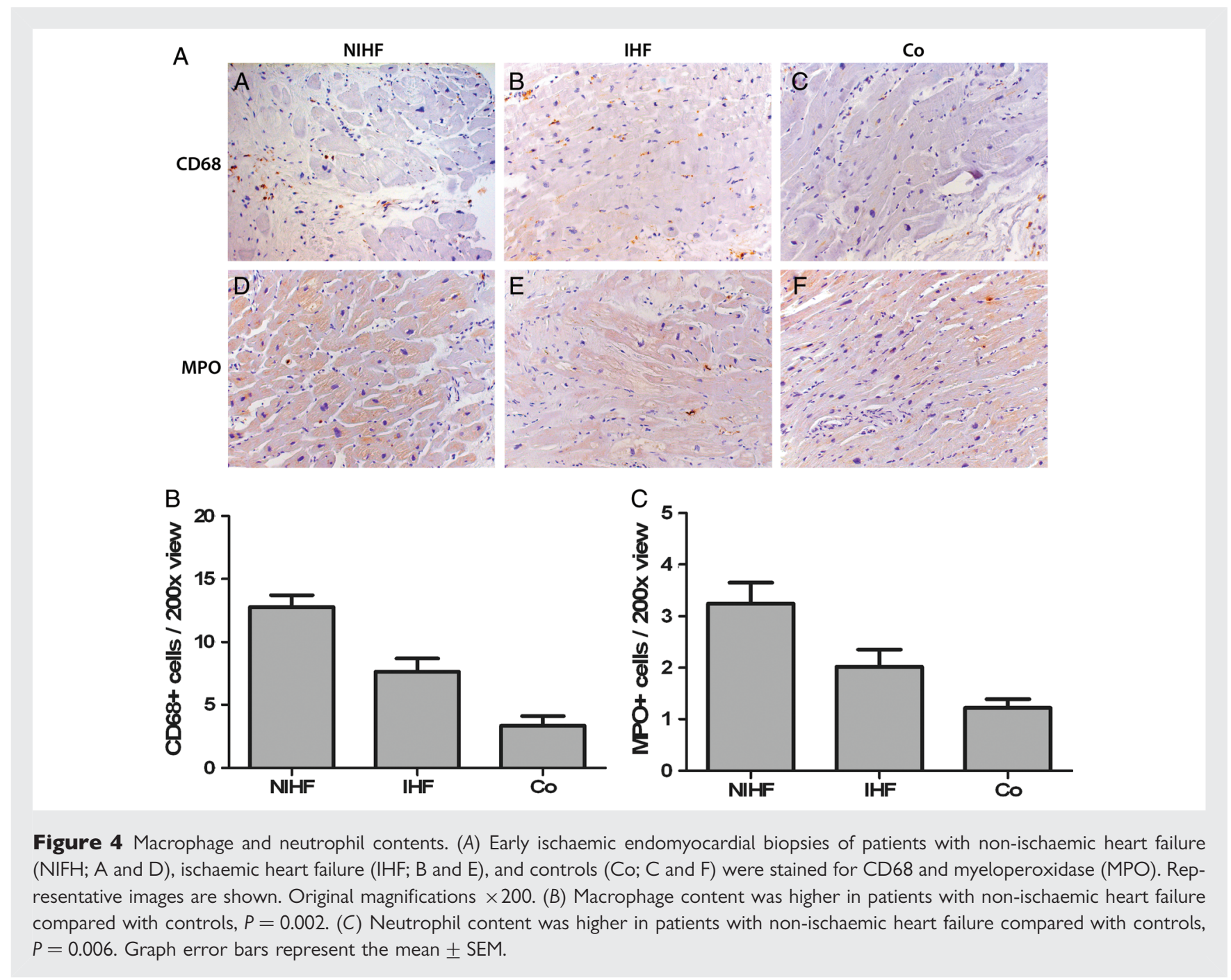

$(P=0.002 ;$ Figure $4 B)$. Also the neutrophil content was 2.7 times higher in patients with non-ischaemic heart failure compared with controls $(P=0.006$; Figure $4 C$ ). T lymphocytes (CD3) were sparsely present in all patient groups, with non-ischaemic heart failure patients showing most $T$ lymphocytes $(P=0.04$, NS). The content of activated leucocytes (HLA-DR) was 3.5 times higher in patients with non-ischaemic heart failure as compared with both ischaemic heart failure and control patients, although not 
significant $(P=0.06)$. No significant changes in macrophage or neutrophil content were observed between the early and late biopsies (Supplementary material, Figure S4).

\section{Discussion}

Since heart failure patients experience various systemic complications post-operatively, we evaluated which inflammatory mediators are released by the myocardium and whether failing hearts differ from non-failing hearts in local inflammatory responses. Besides the expected systemic inflammatory response in all patients, our results show that patients with pre-existing heart failure have a different and more pronounced myocardial inflammatory response after cardiac surgery than controls. Myocardial IL-6 and MCP-1 were released in both non-ischaemic and ischaemic heart failure patients. Furthermore, IL-10, G-CSF, and IL-18 were released in patients with non-ischaemic heart failure, whereas patients with ischaemic heart failure showed a release of VEGF. This myocardial release of various inflammatory mediators may cause systemic complications with a resultant increase in morbidity and mortality.

\section{Release of inflammatory mediators}

Baseline values of the inflammatory markers were not statistically different, although a trend was observed for higher levels in patients with pre-existing heart failure. Various previous studies showed higher cytokine values in patients with heart failure. ${ }^{7,8}$ However, the opposite has been described as well by Vanderheyden et al. ${ }^{9}$ They showed a higher TNF- $\alpha$ and IL- 6 expression in patients with aortic stenosis with normal ejection fraction compared with patients with dilated cardiomyopathy.

In our study, a difference in the myocardial inflammatory response between the two most common types of heart failure was observed, comparing non-ischaemic and ischaemic heart failure patients. Patients with non-ischaemic heart failure showed a more diverse and pronounced release of inflammatory mediators than patients with ischaemic heart failure. The myocardial inflammatory response is, however, specific for patients with heart failure, as controls did not show a myocardial release of inflammatory mediators. This may indicate that controls are able to cope with the stress associated with tissue reperfusion.

\section{Mechanisms of the myocardial inflammatory response}

Mechanisms underlying the observed myocardial inflammatory response in patients with heart failure were analysed in more detail. Since troponin $T$ values were similar between the groups, passive secretion of inflammatory mediators via myocardial damage is unlikely to be a possible cause of the differences. Secondly, similar expression of several inflammatory mediators was observed during ischaemia between the groups. This suggests that the release of inflammatory mediators from pre-stored pools or circulating cells may be responsible for the myocardial response in heart failure patients. Moreover, no cellular activation by means of NF- $\mathrm{kB}$ activity was observed during ischaemia. NF- $\mathrm{B}$ activity was already high at baseline in patients with ischaemic heart failure, which might explain why NF- $\mathrm{KB}$ activity could not increase further during cardioplegic arrest. A more likely explanation for the myocardial release of inflammatory mediators is the observed difference in tissue content of inflammatory cells. Macrophages and neutrophils can release inflammatory mediators such as IL-6, IL-10, IL-18, G-CSF, and MCP-1, ${ }^{10,11}$ which were found to be elevated in our study. Since no changes in transcription of these cytokines were observed and that is likely to take longer, degranulation of prestored cytokine pools is the likeliest explanation. ${ }^{12,13}$ The postreperfusion accumulation and activation of neutrophils in the postischaemic myocardium in the first hours after initiation of myocardial reperfusion have been described in animals. ${ }^{14}$ However, we observed increased amounts of infiltrated neutrophils and macrophages already during early ischaemia. This may suggest the preischaemic presence of higher amounts of inflammatory cells in the failing heart. During reperfusion, these tissue inflammatory cells and their released chemoattractants may amplify the influx of more inflammatory cells into the myocardium, thereby contributing to damage.

\section{Clinical implications}

It is evident that the clinical prognosis after cardiac surgery is influenced by the balance between pro- and anti-inflammatory mediators. Elevated levels of various pro-inflammatory cytokines are associated with adverse events after cardiac surgery. ${ }^{15}$ For example, high circulating IL-6 levels correlate with hepatic and renal dysfunction. ${ }^{16}$ It has been shown that IL-6, IL-8, and MCP-1 showed higher peaks and persisted longer in patients with a complicated course after cardiac surgery in comparison with patients with an uneventful recovery. ${ }^{10}$ In our study, no significant correlations were found between any of the measured inflammatory mediators and intensive care unit or hospital stay.

The increased inflammatory cell content and release of inflammatory mediators in patients with heart failure stresses the potential of pre-operative anti-inflammatory therapy to attenuate the inflammatory response post-operatively. This could contribute to fewer systemic complications and therefore to a more widespread use of cardiac surgical procedures in patients with heart failure. Yet, clinical trials of anti-inflammatory therapies have failed to show a benefit in patients with heart failure. ${ }^{17}$ We should therefore be cautious when intervening with cytokine pathways. In addition to the detrimental role of inflammatory mediators, some also appear to have protective effects depending on the presence of responsive cells, the timing, and the combination of released cytokines. For example, both receptors of TNF have disparate and opposing effects in mice with heart failure. ${ }^{18}$ TNFR1 exacerbates and TNFR2 ameliorates NF-кB activation and inflammation. In renal I/R injury, it has been shown that the role of IL-6 is not straightforward either. ${ }^{19}$ A renal release of IL-6 was observed upon reperfusion in human kidney transplantation, but neutralization of IL-6 in mice undergoing renal I/R injury resulted in aggravation of tissue injury. Therefore, further clinical studies are needed to determine the possibilities of pre-operative anti-inflammatory therapy in patients with heart failure undergoing cardiac surgery. To stress the multifactorial approach of treating patients with heart failure even more, bone marrow dysfunction due to inflammation ${ }^{20}$, renal insufficiency, and haemodilution also plays 
a major role in addition to myocardial and systemic inflammatory responses.

\section{Limitations}

Since the aim of this study was to identify differences in the release of inflammatory mediators between the groups instead of correlating findings to clinical outcome, small patient numbers were sufficient. However, this small sample size might have limited the detection of minor differences (type II errors). The three patient groups are inherently heterogeneous regarding clinical characteristics. No adjustment for these inevitable differences were made. One might consider the collection of pre-reperfusion biopsies only as a limitation. However, a post-reperfusion biopsy could not be collected since most of the included heart failure patients were haemodynamically unstable in the early reperfusion phase. Lifting the heart, to take a biopsy, was considered unsafe. However, human vital myocardial tissue is not often studied since most previous studies used post-mortem biopsies. Moreover, there were also intergroup differences in aortic cross-clamp times. However, no strong relationships between aortic cross-clamp times and plasma inflammatory mediators were observed. In addition, patients with non-ischaemic heart failure had the shortest aortic cross-clamp times, but showed the most diverse release of inflammatory mediators upon reperfusion. Finally, our explorative study was not designed to evaluate inflammatory markers in relation to clinical endpoints. Larger, preferably multicentre, studies are therefore essential.

\section{Conclusions}

Patients with heart failure show a clear myocardial inflammatory response upon reperfusion. This can be explained by degranulation of pre-operatively infiltrated inflammatory cells in myocardial tissue of failing hearts. In contrast, results in control patients indicate that they can better withstand cardioplegic arrest and subsequent reperfusion without a myocardial release of inflammatory mediators.

\section{Supplementary material}

Supplementary material is available at European Journal of Heart Failure online.

\section{Acknowledgements}

The authors gratefully acknowledge Miranda Klerk, Adri Mulder, and Annie jie for the immunohistochemical stainings, RNA extraction and mRNA analysis, and NF- $\mathrm{kB}$ experiments, respectively. Theo Stijnen is thanked for his statistical assistance. Professor Paulus is gratefully acknowledged for critical reading of the manuscript.

\section{Funding}

The Netherlands Heart Foundation [grant number 2007B150 to R.K.].

Conflict of interest: None declared.

\section{References}

1. Kortekaas KA, Lindeman JH, Versteegh MI, Stijnen T, Dion RA, Klautz RJ. Preexisting heart failure is an underestimated risk factor in cardiac surgery. Neth Heart J 2012;20:202-207.

2. Dewald O, Frangogiannis NG, Zoerlein M, Duerr GD, Klemm C, Knuefermann P, Taffet G, Michael LH, Crapo JD, Welz A, Entman ML. Development of murine ischemic cardiomyopathy is associated with a transient inflammatory reaction and depends on reactive oxygen species. Proc Natl Acad Sci USA 2003;100: 2700-2705.

3. Liu P, Hock CE, Nagele R, Wong PY. Formation of nitric oxide, superoxide, and peroxynitrite in myocardial ischemia-reperfusion injury in rats. Am J Physiol 1997; 272:2327-2336.

4. Luo X, Cai H, Ni J, Bhindi R, Lowe HC, Chesterman CN, Khachigian LM. c-Jun DNAzymes inhibit myocardial inflammation, ROS generation, infarct size, and improve cardiac function after ischemia-reperfusion injury. Arterioscler Thromb Vasc Biol 2009;29:1836-1842.

5. Chuang ML, Hibberd MG, Salton CJ, Beaudin RA, Riley MF, Parker RA, Douglas PS, Manning WJ. Importance of imaging method over imaging modality in noninvasive determination of left ventricular volumes and ejection fraction: assessment by two- and three-dimensional echocardiography and magnetic resonance imaging. J Am Coll Cardiol 2000;35:477-484.

6. Kleemann R, van Erk M, Verschuren L, van den Hoek AM, Koek M, Wielinga PY, Jie A, Pellis L, Bobeldijk-Pastorova I, Kelder T, Toet K, Wopereis S, Cnubben N, Evelo C, van Ommen B, Kooistra T. Time-resolved and tissue-specific systems analysis of the pathogenesis of insulin resistance. PLoS One 2010;5:8817-8833.

7. El-Menyar AA. Cytokines and myocardial dysfunction: state of the art. J Cardiac Fail 2008;14:61-74.

8. Yndestad A, Holm AM, Muller F, Simonsen S, Froland SS, Gullestad L, Aukrust P. Enhanced expression of inflammatory cytokines and activation markers in T-cells from patients with chronic heart failure. Cardiovasc Res 2003;60:141-146.

9. Vanderheyden M, Paulus WJ, Voss M, Knuefermann P, Sivasubramanian N, Mann D, Baumgarten G. Myocardial cytokine gene expression is higher in aortic stenosis than in idiopathic dilated cardiomyopathy. Heart 2005;91: 926-931.

10. Fujiwara T, Seo N, Murayama T, Hirata S, Kawahito K, Kawakami M. Transient rise in serum cytokines during coronary artery bypass graft surgery. Eur Cytokine Netw 1997;8:61-66

11. Cavaillon JM. Cytokines and macrophages. Biomed Pharmacother 1994;48: 445-453.

12. Hellweg CE, Baumstark-Khan C, Horneck G. Generation of stably transfected mammalian cell lines as fluorescent screening assay for NF-kappaB activationdependent gene expression. J Biomol Screen 2003;8:511-521.

13. Li X, Zhao X, Fang Y, Jiang X, Duong T, Fan C, Huang CC, Kain SR. Generation of destabilized green fluorescent protein as a transcription reporter. J Biol Chem 1998;273:34970-34975.

14. Rashid MA, William-Olsson G. Are leukocytosis and lipid peroxidation involved in ischemic or reperfusion injury in cardiac surgery? Thorac Cardiovasc Surg 1991;39: 193-195.

15. Hirai S, Sueda T, Orihashi K, Watari M, Okada K. Kinetics of pro-inflammatory cytokines release in cardiac surgery with cardiopulmonary bypass. Jpn J Thorac Cardiovasc Surg 2001;49:216-219.

16. Kawamura T, Wakusawa R, Okada K, Inada S. Elevation of cytokines during open heart surgery with cardiopulmonary bypass: participation of interleukin 8 and 6 in reperfusion injury. Can J Anaesth 1993;40:1016-1021.

17. Heymans S, Hirsch E, Anker SD, Aukrust P, Balligand JL, Cohen-Tervaert JW, Drexler H, Filippatos G, Felix SB, Gullestad L, Hilfiker-Kleiner D, Janssens S, Latini R, Neubauer G, Paulus WJ, Pieske B, Ponikowski P, Schroen B, Schultheiss HP, Tschope C, Van Bilsen M, Zannad F, McMurray J, Shah AM. Inflammation as a therapeutic target in heart failure? A scientific statement from the Translational Research Committee of the Heart Failure Association of the European Society of Cardiology. Eur J Heart Fail 2009;11:119-129.

18. Hamid T, Gu Y, Ortines RV, Bhattacharya C, Wang G, Xuan YT, Prabhu SD. Divergent tumor necrosis factor receptor-related remodeling responses in heart failure: role of nuclear factor-kappaB and inflammatory activation. Circulation 2009;119:1386-1397.

19. de Vries DK, Lindeman JH, Tsikas D, de Heer E, Roos A, de Fijter JW, Baranski AG, van Pelt J, Schaapherder AF. Early renal ischemia-reperfusion injury in humans is dominated by IL-6 release from the allograft. Am J Transplant 2009;9:1574-1584.

20. Westenbrink BD, Voors AA, de Boer RA, Schuringa J], Klinkenberg T, van der Harst P, Vellenga E, van Veldhuisen DJ, van Gilst WH. Bone marrow dysfunction in chronic heart failure patients. Eur J Heart Fail 2010;12:676-684. 